\title{
Materanal and neonatal outcomes of triplet gestation in a tertiary care centre: a retrospective study
}

\author{
Neeraj Sharma, Deepika Kumari*, Sonal Prasad, Akanksha Srivastava
}

Department of Obstetrics and Gynecology, Dr. Baba Saheb Ambedkar Hospital, Rohini, Delhi, India

Received: 05 August 2019

Revised: 25 September 2019

Accepted: 30 September 2019

\section{*Correspondence:}

Dr. Deepika Kumari,

E-mail: deepikadmch2011@gmail.com

Copyright: () the author(s), publisher and licensee Medip Academy. This is an open-access article distributed under the terms of the Creative Commons Attribution Non-Commercial License, which permits unrestricted non-commercial use, distribution, and reproduction in any medium, provided the original work is properly cited.

\begin{abstract}
Background: A significant rise in triplet pregnancy rate has occurred recently. This rise is of concern, as these infants are frequently reported as a risk factor of adverse outcome. The aim of this study was to retrospectively study the outcomes of triplet births.

Methods: A retrospective study was undertaken to analyze the clinical data of all patients with triplet gestation admitted to the labour room of Dr. Baba Saheb Ambedkar Hospital, Delhi to study the maternal and fetal outcomes. Authors retrospectively observed and analyzed the database to examine triplet gestations delivered between January 2014 and December 2018.

Results: Over the five-year study period, there were total 44,011 deliveries. Out of these, there were $35(0.079 \%)$ triplet pregnancies. Of all the triplet pregnancies studied $80 \%$ resulted from ovulation induction and assisted reproductive technology. The mean gestational age at delivery was $31.6 \pm 3.0$ weeks, and the mean birth weight was $1,594 \pm 460 \mathrm{gm}$. The most common maternal complications were Preterm labour in 32 pregnancies (92\%), anemia in $17(49 \%)$, pre-eclampsia in $11(31 \%)$, post-partum hemorrhage in $8(22 \%)$. Of the total deliveries neonatal complications included Respiratory distress syndrome in 44 (42.2\%), Hyperbilirubinemia in 41 (39\%), Intrauterine growth restriction in $19(18.1 \%)$. The perinatal mortality rate was $10.5 \%$.

Conclusions: Higher order pregnancies are associated with maternal and neonatal complications. These high risk women need more care and the neonates require intensive care and monitoring after birth, most commonly due to prematurity and low birth weight.
\end{abstract}

Keywords: Morbidity, Perinatal mortality, Preterm births, Triplet pregnancies

\section{INTRODUCTION}

Over the last 20 years, there has been a $400 \%$ rise in the rate of higher-order multiple births. ${ }^{1}$ Two important factors associated with this are tendency towards increased maternal age, which may be associated with increased rates of spontaneous multiple births, and increasing widespread availability of medical assistance for conception such as ovulation induction and assisted reproductive technologies (ART) most commonly in vitro fertilization (IVF). ${ }^{2}$
Multiple pregnancy is associated with higher risks for both mother and neonate. Women with multiple pregnancies have an increased risk of miscarriage, anemia, hypertensive disorders, hemorrhage, need for operative deliveries, and postpartum illness. Neonatal complications include Respiratory distress syndrome (RDS), bronchopulmonary dysplasia (BPD), chronic lung disease, Retinopathy of prematurity (ROP), Pneumothorax, Patent ductus arteriosus (PDA), necrotizing enterocolitis (NEC), Intraventricular hemorrhage, hydrocephalous, congenital anomalies and 
Perinatal mortality. Preterm delivery with low birth weight is the most common complication of triplet pregnancy, with approximately $75 \%$ to $100 \%$ of the triplets being born prematurely. ${ }^{3}$

Neonatal complications are mainly related to prematurity and intrauterine growth restrictions. The significant higher preterm delivery rates in multiple pregnancies imply the increased demand for specialist neonatal resources. Due to increased risk of complications, women with multiple pregnancies require more monitoring, additional calories and proteins, and increased ANC visits at hospital during their pregnancy as compared to singleton pregnancies. ${ }^{4}$

The combination of the growing number of triplet pregnancies and the potential for problems facilitated the requirement and importance of the present study. The role of infertility treatment methods practiced results in high incidence of multiple births and thus, this lead to the adoption of a more restrictive policy of reproductive medicine by many countries, setting an example for the highest limit of embryo transfer at two. ${ }^{5}$ In countries following such policy, this has shown to be effective, as demonstrated by Robert et al, who discovered no significant rise in higher order pregnancies in New South Wales, Australia during the last decade.,

The reduction in multiple pregnancies is a widespread therapy to reduce the risk of prematurity and adverse outcome of the neonates in higher order pregnancies. ${ }^{7}$ Selective fetal reduction is a method that has been widely performed over the last 15 years to reduce the risk of complications associated with multiple gestation, and correspondingly improve the pregnancy outcome. ${ }^{8}$ Many investigators have compared the outcomes among the triplet with reduced and non-reduced pregnancies with many conflicting results. Studies in early 1990s suggested that reduction of triplet pregnancies improved outcomes. However, the recent studies have failed to demonstrate adverse outcomes in triplet pregnancies when compared with reduced and non-reduced twins. ${ }^{9-11}$ Multifetal pregnancy reduction is ethically problematic and also difficult for patients to accept and therefore should not be included a part of routine infertility treatment planning. ${ }^{7}$

\section{METHODS}

In this study, which was conducted from the beginning of 2014 to the end of 2018, the data of 35 triplet pregnancies delivered at a Tertiary Obstetric Referral Centre, Dr. Baba Saheb Ambedkar Hospital, Delhi were used. For this study, triplet pregnancies were defined as a pregnancy that began at 20 weeks period of gestation, and were registered at Dr. Baba Saheb Ambedkar Hospital, Delhi. In this study, the number of triplets reduced to twins were not included. Data were collected by design sheets, including maternal and fetal information according to the documents of each mother and neonates by a resident. Exclusion criteria included: incomplete documents of mother and neonates and induced abortions of higher order multiple pregnancies. Finally, maternal and neonatal data were analyzed.

\section{Statistical analysis}

For the comparison of quantitative variables of triplet pregnancies, independent t-test (for the normally distributed variables) and Mann - Whitney test (for variables without normal distribution) were used, and for the comparison quantitative variables, chi-square test (for variables with chi-square test assumptions) and Fisher's exact test (for variables without chi-square assumptions) were used.

\section{RESULTS}

Between January 2014 and December 2018, out of total 44011 deliveries, 35 sets of triplets (105 neonates) were born at Dr. Baba Saheb Ambedkar Hospital, Delhi. Complete data were available for the births.

With regards to the average age, the sample was quite young (average age was of 28) and the average body mass indices based on the standard guidelines corresponded with the overweight group (mean 28). The average gestational age for termination was $31.6 \pm 3$ weeks, the average weight of the first neonate to the third was $1500 \mathrm{gm}$. The mean gestational age and birth weight, respectively in the triplet pregnancy was 33 weeks and 4 days and $1557 \mathrm{gm}$. The Apgar score of all neonates in both measurements (first and fifth minute) was 8 . The mean duration of hospitalization in surviving neonates was 22-26 days.

This study demonstrated that $99 \%$ of neonates were under 37 weeks (preterm) of gestation, among which $72 \%$ were under 34 weeks of gestation, and $16.3 \%$ were under 28 weeks of gestation. The weight of the neonates at birth in the first neonate in $97 \%$, second neonate in $96.3 \%$, and the third neonate in $97 \%$ was below 2500 gm. Thus, the percentage of neonates between $2500 \mathrm{gm}, 2000 \mathrm{gm}$ and $1500 \mathrm{gm}$ (low birth weight) were respectively $45.5 \%$, $44.6 \%$, and $36.6 \%$.

The majority of the women were without any history of stillbirth, though they had history of infertility treatment, but no history of any abortion (medical or spontaneous), ectopic pregnancy and preterm birth. $80.5 \%$ of women in this study had a history of infertility treatment, and the majority (80\%) conceived with assisted reproductive technology (ART). None of the women had experience of smoking, drug or alcohol abuse.

The experience of Cerclage was surveyed in only 2 women. The use of progesterone was positive in majority of the subjects $(56.6 \%)$, among consumers the intravaginal form was more common than the systemic form of progesterone. Majority $(82 \%)$ of the women had 
received at least one to two courses of Dexamethasone for lung maturity of the fetus.

The results of this study demonstrated that the majority of women $(64 \%)$ experienced the complications $92 \%$ of women had pre-term labour, $49 \%$ suffered from anemia, $22.8 \%$ had uterine atony causing post-partum hemorrhage of which one case led to Hysterectomy, $31 \%$ of the women had preeclampsia of which two cases led to HELLP syndrome, $10 \%$ had gestational diabetes, there was $8 \%$ blood transfusion after uterine atony and postpartum hemorrhage.

Preterm labour without rupture of membrane (ROM) was observed in 19 cases of triplet pregnancies (56\%), and premature rupture of membranes (PROM) was observed in 10 cases of triplet pregnancies $(29.5 \%)$.

Fetal complications were observed in $68 \%$ neonates Respiratory distress syndrome (RDS) was observed in 44 neonates $(42.2 \%)$, hyperbilirubinemia in 41 neonates $(39.0 \%)$, intrauterine growth restriction was observed among 19 neonates $(18.1 \%)$, perinatal mortality rate was $10.5 \%$ there were two cases of intrauterine deaths and 9 cases of deaths after birth (total 11 neonates ), congenital anomalies in 4 neonates, most common being congenital heart anomaly in two neonates, cleft lip in one neonate, and tracheoesophageal fistula (TEF) in one neonate, patent ductus arteriosus (PDA) in 3 neonates $(2.9 \%)$, sepsis, retinopathy of prematurity (ROP) was observed in $1(0.95 \%)$ of neonates, hydrocephalus was observed in one $(0.95 \%)$ neonate.

In this study one pregnancy was associated with chorioamnionitis, two neonates had acidosis at birth time ( $\mathrm{pH}$ less than 7), and there were no cases of intraventricular hemorrhage, periventricular leukomalacia, advanced retinopathy of prematurity or cerebral palsy.

To compare the quantitative variables of women with triplet deliveries, independent t-test (for normally distributed variables) and Mann - Whittney test (for the variables without normal distribution) were used. The result of the tests was - The mean age of the women in triplet deliveries were significantly lower. The mean length of hospital stay was 23 days, with a median of 19 days. Triplet survival to hospital discharge rate was improved as compared to earlier records. Although, the high perinatal mortality rate of $10.5 \%$ is of concern.

\section{DISCUSSION}

According to the recently published National vital statistics for triplets, the national average gestational age for triplet pregnancy is 32.2 weeks, with $34.6 \%$ of births occurring between 34 to 36 weeks of gestation. ${ }^{12}$ The national average birth weight for triplets is $1698 \mathrm{gm}$. The present study demonstrates comparable results.
Previous studies suggest that higher-order multiples are more likely to have discordant birth weights than twins and singletons. These earlier studies reported discordancy rates of $48 \%$ and $54 \%$ for triplets, as compared to 13 and $15 \%$ for twins. Discordancy rates were $17 \%$ in the current series, a rate similar to that seen in twins in the previous studies. Improved obstetric management of triplet pregnancies may be one of the factor contributing to the observed decrease in discordancy. Congenital abnormalities occurred in $4(3.81 \%)$ of triplets in this series and most common was congenital heart defects. This rates compared favorably with a $4.8 \%$ rate of major congenital abnormalities reported in 1998 and adds further weight to the supposition that triplets are not at risk of adverse outcomes. ${ }^{13}$

This series demonstrates very low rates of morbidity associated with triplet births. There were no reports of intraventricular hemorrhage, advanced retinopathy of prematurity, only $3 \%$ for chronic lung disease and no case of necrotizing enterocolitis. Previous work has reported similar frequencies of bronchopulmonary dysplasia, necrotizing enterocolitis, intraventricular hemorrhage and periventricular leucomalacia between twins and triplets, but it suggested that triplets had a statistically significant increased risk of retinopathy of prematurity. ${ }^{13}$ Increased clinical surveillance and heighted awareness may have contributed to lack of excess of retinopathy in this current study. Length of hospital stay is reported to be longer for triplets, with one study describing a mean hospital stay of 51 days for triplets born at 31 weeks. ${ }^{14}$ The current study shows shorter hospital stay of 22-26 days, such difference is due to variation in discharge policy over time, as well as improvements in the neonatal care.

In the current series, $96 \%$ of triplets survived to discharge. Over the last 20 years, survival in higher-order pregnancies have gradually improved. For example, survival rates in the late 1980s were reported to be $79 \%$, and the most recent National Statistics including births up to 1994 reported $90.6 \%$ of triplets surviving. ${ }^{15,16}$ This improvement is also confirmed in other cohort studies quoting survival rates of 94 to $97 \%$ (21-31\%).

During the early 1990s studies suggested benefits in neonatal outcome and survival rates if triplet pregnancies were reduced to twin pregnancies. For example, one series demonstrated the improvement of neonatal survival from 79 to $94 \%$ if triplet pregnancies that were reduced to twins. However, this perceived benefit in neonatal survival and has not been maintained in studies published recently. In a study reported in 2000, there was no difference in neonatal survival when 81 sets of triplets were compared with 46 sets of triplets reduced to twins. ${ }^{17}$ In addition, 1999 study comparing 25 sets of triplets with 19 sets of reduced twins has reported no difference in the overall neonatal survival. ${ }^{18}$ However, in our institution fetal reduction facilities are not available so, the comparison could not be made. Nevertheless, the findings 
regarding maternal and fetal outcomes are consistent with the recently published literature.

\section{CONCLUSION}

This large contemporary case series of triplet pregnancy demonstrates excellent survival rates with very low associated morbidity. Given our data and several other reports of such improvements, the need to offer selective fetal reduction to the parents expecting triplets should be re-considered. This study showed that rate of development of respiratory distress syndrome were lower in neonates whose mother received steroid in antenatal period as compared to mothers who did not receive steroid, due to higher rates of prematurity in fetus. There should be further studies on various maternal and fetal complications due to increasing incidence of triplet pregnancies, which also accounts for the need for special maternal, fetal, and neonatal care. Also, to overcome the controversies related to Assisted reproductive techniques (ART), procedures of cervical cerclage and their use, further studies can be helpful.

This study shall be of help to obstetricians and health care workers who need to spread awareness regarding routine Antenatal visits, adequate nutrition, needful investigations, and administration of antenatal steroids among the women with higher order pregnancies. This will be a major step in improving most common maternal complications such as anemia, intrauterine growth restriction, and to overcome the neonatal complications of respiratory distress syndrome and reduce the incidences of perinatal mortality.

\section{Funding: No funding sources}

Conflict of interest: None declared

Ethical approval: The study was approved by the Institutional Ethics Committee

\section{REFERENCES}

1. Centers for Disease Control and Prevention. Contribution of assisted reproductive technology and ovulation - inducing drugs to triplet and higher-order multiple births - United States, 1980-1997. JAMA. 2000;284:299-300.

2. Mirzamoradi M, Heidar Z, Saleh M. Fetomaternal outcome in triplet and quadruplet pregnancies: a retrospective study. Int $\mathbf{J}$ Med Res Health Sci. 2017;6(6):166-70.

3. Albrecht JL, Tomich PG. The maternal and neonatal outcome of triplet gestations. Am J Obstet Gynecol. 1996;174(5):1551-6.

4. Platt MP, Glinianaia SV, Rankin J, Wright C, Renwick M. Northern Region multiple pregnancy register steering group. The North of England multiple pregnancy register: five-year results of data collection. Twin Res Human Genetics. 2006;9(6):913-8.
5. Garite TJ, Clark RH, Elliott JP, Thorp JA, Pediatrix/obstetrix perinatal research group. Twins and triplets: the effect of plurality and growth on neonatal outcome compared with singleton infants. Am J Obstet Gynecol. 2004;191(3):700-7.

6. Ho ML, Chen JY, Ling UP, Chen JH, Huang CM, Chang CC, et al. Changing epidemiology of triplet pregnancy: etiology and outcome over twelve years. Am J Perinatol. 1996;13(05):269-75.

7. Devine PC, Malone FD, Athanassiou A, Harvey-Wilkes K, D'Alton ME. Maternal and neonatal outcome of 100 consecutive triplet pregnancies. Am J Perinatol. 2001;18(04):225-36.

8. Evans MI, Berkowitz RL, Wapner RJ. Improvement in outcomes of multifetal pregnancy reduction with increased experience. Am J Obstet Gynecol. 2001;184:97-103.

9. Antsaklis AJ, Drakakis P, Vlazakis GP, Michalas S. Reduction of multifetal pregnancies to wins does not increase obstetric or perinatal risks. Hum Reprod. 1999; 14:1338-40.

10. Bell MJ, Ternberg JL, Feigin RD. Neonatal necrotizing enterocolitis. Therapeutic decisions based upon clinical staging. Ann Surg. 1978;187:1-7.

11. Papile LA, Burstein R. Incidence and evolution of subependymal and intraventricular hemorrhage: a study of infants with birth weights less than $1500 \mathrm{~g}$. J Pediatr. 1978;529-34.

12. Tarter JG, Khoury A, Barton JR. Demographic and obstetric factors influencing pregnancy outcomes in twin gestations. Am J Obstet Gynecol. 2002;18:910-2.

13. Kaufman GE, Malone FD, Harvey-Wilkes KB. Neonatal morbidity and mortality associated with triplet pregnancy. Obstet Gynecol. 1998;91:342-8.

14. Suri K, Bhandari V, Lerer T, Rosenkrantz TS, Hussain $\mathrm{N}$. Morbidity and mortality of preterm twins and higher-order multiple births. J Perinatol. 2001;21:293-9.

15. Macones GA, Shemmer G, Pritis E. Multifetal reduction of triplets to twins improves perinatal outcome. Am J Obstet Gynecol. 1993;169:982-6.

16. Martin JA, MacDorman MF, Mathew TJ. Triplet births: Trends and Outcomes, 1971-94. National Vital Statistics Reports. Series. 19997;21(55):7.

17. Leondires MP, Ernst SD, Miller BT, Scott Jr RT, Triplets: outcomes of expectant management versus multifetal reduction for 127 pregnancies. Am journal Obstet Gynecol. 2000;183(2):454-9.

18. Angel JL, Kalter CS, Morales WJ, Rasmussen C, Caron L. Aggressive perinatal care for high-order multiple gestations: Does good perinatal outcome justify aggressive assisted reproductive techniques?. Am J Obstet Gynecol 1999; 181:253-9.

Cite this article as: Sharma N, Kumari D, Prasad S, Srivastava A. Materanal and neonatal outcomes of triplet gestation in a tertiary care centre: a retrospective study. Int J Reprod Contracept Obstet Gynecol 2019;8:4453-6. 